\title{
An overlooked combination in treatment: addiction and social anxiety disorder comorbidity
}

\author{
Cuneyt Evren ${ }^{1}$
}

'Bakirkoy Training and Research Hospital for Psychiatry Neurology and Neurosurgery, Research, Treatment and Training Center for Alcohol and Substance Dependence (AMATEM), Istanbul - Turkey

The majority of studies of social anxiety disorder (SAD) and alcohol and substance use disorder (ASUD) comorbidity focus on alcohol, though there is also research examining cannabis, nicotine, and other dependencies. It is generally accepted that the risk for substance use is greater in patients diagnosed with SAD. Comorbid SAD and ASUD is clinically important, as it associated with higher morbidity, lower treatment response, and less treatment-seeking behavior $(1,2)$.

It is generally suggested that individuals with anxiety disorders start using substances to cope with their symptoms (self-medication). However, substance use may also be the cause of or create a predisposition to anxiety disorders due to changes in the biology of the brain $(1,2)$.

The early onset of SAD, combined with the later onset of substance use, explains the ordinal relationship observed. It has been reported that among $87 \%$ of those diagnosed with alcohol use disorder (AUD) and SAD, SAD were diagnosed before AUD (3) and AUD developed secondary to SAD 4 times more than other anxiety disorders (4). Similarly, it has been reported that among individuals with cannabis use disorder and SAD, SAD developed first in 4 of 5 individuals (5). SAD has also been found to have developed before opioid use disorder (6). However, the onset of SAD before ASUD does not fully explain the causality relationship between them, particularly since SAD is a disease that typically begins in early adolescence (1).
In some individuals, substance use may lead to increased social anxiety. The results of the US National Comorbidity Survey showed that although SAD is a precursor to AUD in most individuals with that comorbidity, AUD may precede SAD in some portion of that group (7). In some individuals, alcohol use to cope with social anxiety (particularly observational fears) can create a vicious circle of increased social anxiety, leading to more alcohol use in the next social interaction (1).

Regardless of the order of occurrence, once SAD and ASUD are present, the 2 diseases have a negative effect each other, contributing to persistence and aggravation. The substance used to alleviate anxiety prompts further ASUD, while anxiety occurring during substance intoxication or deprivation can cause the aggravation of symptoms (1).

Diagnosing Comorbidity: One of the methods that can be used in SAD screening is to ask the patient the following questions:

- Have you ever noticed that you are avoiding social situations or activities?

- Do you feel scared or embarrassed in social situations?

If the response to one of the two questions is yes, a detailed interview is recommended for SAD (1). The point to be kept in mind is that even if the SAD criteria are not met, addressing subthreshold SAD symptoms may benefit an individual with ASUD during the treatment process. Studies show that individuals with subthreshold SAD are also at risk for ASUD (8).

How to cite this article: Evren C. An overlooked combination in treatment: addiction and social anxiety disorder comorbidity. Dusunen Adam The Journal of Psychiatry and Neurological Sciences 2020;33:331-333.

Correspondence: Cuneyt Evren, Bakirkoy Training and Research Hospital for Psychiatry Neurology and Neurosurgery, Research, Treatment and Training Center for Alcohol and Substance Dependence (AMATEM), Istanbul - Turkey

Phone: +90 21254365 65-21 11 E-mail: cuneytevren@yahoo.com 
Since anxiety symptoms can be seen during the withdrawal period of many substances as well as during intoxication with some substances, it is recommended that the withdrawal period be completed before patients are diagnosed with an additional anxiety disorder and to further evaluate the patient afterwards $(1,2)$.

Factors Associated with Comorbidity: Anxiety can include a fear of somatic symptoms, such as an increased heart rate or muscle tension, as these symptoms may lead to negative physical, mental, or social consequences (1). People with a high sensitivity to anxiety may have a predisposition for anxiety disorders, including SAD (1).

The importance of the relationship between a belief in individuals with SAD that alcohol consumption will create a desirable effect and alcohol use has been highlighted in a review $(1,2,9)$. It has been observed that people with SAD and AUD had more positive expectations (such as decreased tension, social assertiveness) about the effect of alcohol than those without AUD and those with lower anxiety levels $(1,2,9)$.

Shy or socially anxious young people are often less likely to use substances or be in social situations where substance use present because their social network is small and they may not have good interpersonal skills $(1,2)$. In addition, young people with social anxiety may avoid substance use in order not to display behaviors that would be considered negative. For example, it has been reported that the expectation of negative behavior related to cannabis use (such as speaking nonsense or acting excited and appearing foolish) may create an inverse relationship between social anxiety and cannabis use $(1,2)$. On the other hand, peer substance use is often influential in terms of starting, quitting, and continuing substance use. Young people with social anxiety may adopt behaviors such as substance use when it is present around them, especially when there is a fear of rejection (1).

When individuals with SAD drink alcohol, they often feel freer to talk to another person and receive more positive social responses. Although people with SAD may face difficulties in social situations, they do want to build close relationships with others. The fact that alcohol or other substances can facilitate that desired result increases the risk of use $(1,2)$.

Shyness and social anxiety can be difficult to evaluate and can have varied impacts on substance use (1). Contradictory results obtained in studies of SAD and substance use can be explained by the fact that the degree of SAD can have different effects on ASUD. Those who have social anxiety that impairs their functionality, that is SAD, are at higher risk for ASUD than others who may only have some social anxiety symptoms $(1,2)$.

The relationship between SAD and ASUD is also influenced by other factors. For example, adverse early childhood events increase the risk of anxiety disorder, which increases the risk of developing ASUD $(1,2)$. It has been reported that individuals with SAD and AUD received less care from their mothers, experienced more negative events in their childhood, and displayed a lower level of cooperation in assessment of character traits (10).

Individuals with comorbid SAD and AUD also have a higher risk of developing another psychiatric disease, especially depression or depressive symptoms $(1,2)$. This makes it more difficult to plan treatment. Furthermore, it is noteworthy that comorbidity increases suicide attempts, suicidal ideation, and suicide planning $(1,2)$.

Treatment: It is important for clinicians to inform patients with SAD who report using alcohol about how to cope with their anxiety and to review the risks. Although AUD may not directly lead to the development of SAD, it is clear that it worsens its course. Therefore, it is also important that patients do not use alcohol during SAD treatment (1).

Comorbidity can impair treatment compliance (2). It may be more difficult for people with social anxiety symptoms and ASUD to participate in and benefit from traditional therapies and related activities, such as group-based treatment that requires active participation. These patients can be unfairly accused of avoiding group therapy and criticized for not adequately participating in their recovery by the treatment team (2).

Comorbid SAD and ASUD often reduces treatment compliance and success for both diseases. Therefore, it is recommended to start simultaneous, integrated treatment and to follow the patient carefully. Unfortunately, the availability of such conditions is limited $(1,2)$.

The first-line pharmacological treatment of SAD is a selective serotonin reuptake inhibitor (SSRI). If no benefit is obtained with an SSRI, venlafaxine, a serotonin noradrenaline inhibitor, may be considered (11). The existing data on safe use of benzodiazepines is currently inadequate, and although it is not contraindicated, the prescription of benzodiazepines should be avoided as much as possible in a patient with SAD who is also using alcohol or other mind- and behavior-altering substances $(1,2)$. 
Generally, cognitive behavioral therapy (CBT) is the first-line treatment, and exposure techniques have been shown to be effective (11). It was reported in a review that psychotherapy focused on ASUD and anxiety disorders did not yield better results than psychotherapy focused on solely ASUD (12). However, a subsequent meta-analysis demonstrated that both CBT and pharmacotherapy had a moderate effect on alcohol and anxiety treatment outcomes in patients in a treatment program for AUD (13).

SAD and ASUD comorbidity may be present more often than recognized in clinical practice, as it is a complex picture and can often be overlooked. Considering that the treatment seeking behaviour is low for both of these disorders, the patients admitted to the hospital for one of these disorders should be investigated by the clinicians for the other.

\section{REFERENCES}

1. Bozkurt M. An overlooked disorder in diagnosis and treatment: substance use disorder and social anxiety disorder: In Evren C. (editor) Comorbidities and special cases in alcohol and substance use disorders. İstanbul: Library of Dusunen Adam The Journal of Psychiatry and Neurological Sciences-5, Yerkure, 2019.

2. Evren C. Social anxiety disorder and alcohol use disorders. Current Approaches in Psychiatry 2010; 2:473-515.

3. Pacek LR, Storr CL, Mojtabai R, Green KM, La Flair LN, Alvanzo AA, et al. Comorbid alcohol dependence and anxiety disorders: a national survey. J Dual Diagn 2013; 9:10.1080/15504263.2013.835164.

4. Boschloo L, Vogelzangs N, Smit JH, van den Brink W, Veltman DJ, Beekman AT, et al. Comorbidity and risk indicators for alcohol use disorders among persons with anxiety and/or depressive disorders: findings from the Netherlands Study of Depression and Anxiety (NESDA). J Affect Disord 2011; 131:233-242.
5. Buckner JD, Heimberg RG, Schneier FR, Liu SM, Wang S, Blanco C. The relationship between cannabis use disorders and social anxiety disorder in the National Epidemiological Study of Alcohol and Related Conditions (NESARC). Drug Alcohol Depend 2012; 124:128-134.

6. Fatséas M, Denis C, Lavie E, Auriacombe M. Relationship between anxiety disorders and opiate dependence--a systematic review of the literature: implications for diagnosis and treatment. J Subst Abuse Treat 2010; 38:220-230.

7. Buckner JD, Timpano KR, Zvolensky MJ, Sachs-Ericsson N, Schmidt NB. Implications of comorbid alcohol dependence among individuals with social anxiety disorder. Depress Anxiety 2008; 25:1028-1037.

8. Crum RM, Pratt LA. Risk of heavy drinking and alcohol use disorders in social phobia: a prospective analysis. Am J Psychiatry 2001; 158:1693-1700.

9. Evren C, Aksoy R, Celik S, Cetin T, Tamar-Gurol D. Relationship of social anxiety severity with drinking motives among male alcohol dependent inpatients. European Psychiatry 2011; 26:35.

10. Rambau S, Forstner AJ, Wegener I, Mücke M, Wissussek CTS, Staufenbiel SM, et al. Childhood adversities, bonding, and personality in social anxiety disorder with alcohol use disorder. Psychiatry Res 2018; 262:295-302.

11. National Institute For Health and Care Excellence (NICE), National Collaborating Centre for Mental Heath. Social anxiety disorder: the NICE guideline on rocognition, assesment and treatment. Leicester: British Psychological Societty, 2013. https:// www.ncbi.nlm.nih.gov/books/NBK266258/pdf/Bookshelf_ NBK266258.pdf Accessed December 15, 2020.

12. Hesse M. Integrated psychological treatment for substance use and co-morbid anxiety or depression vs. treatment for substance use alone. A systematic review of the published literature. BMC Psychiatry 2009; 9:6.

13. Hobbs JD, Kushner MG, Lee SS, Reardon SM, Maurer EW. Metaanalysis of supplemental treatment for depressive and anxiety disorders in patients being treated for alcohol dependence. Am J Addict 2011; 20:319-329. 